\title{
An Accuracy-Improved Fixed-Width Booth Multiplier Enabling Bit-Width Adaptive Truncation Error Compensation
}

\author{
Song-Nien Tang *, Jen-Chien Liao, Chen-Kai Chiu, Pei-Tong Ku and Yen-Shuo Chen
}

Chiu, C.-K.; Ku, P.-T.; Chen, Y.-S. An Accuracy-Improved Fixed-Width Booth Multiplier Enabling Bit-Width Adaptive Truncation Error Compensation. Electronics 2021, 10, 2511. https://doi.org/10.3390/ electronics10202511

Academic Editor: Spiros Nikolaidis

Received: 26 August 2021

Accepted: 13 October 2021

Published: 15 October 2021

Publisher's Note: MDPI stays neutral with regard to jurisdictional claims in published maps and institutional affiliations.

Information and Computer Engineering Department, Chung Yuan Christian University, Taoyuan 32023, Taiwan; liaojack1233@gmail.com (J.-C.L.); nike7014@gmail.com (C.-K.C.); jin0917362909@gmail.com (P.-T.K.); danie10319@livemail.tw (Y.-S.C.)

* Correspondence: sntang@ice.cycu.edu.tw; Tel.: +886-3-26654731

\begin{abstract}
Fixed-width Booth multipliers (FWBMs) generate a product with the same bit width as the operand and have been extensively employed in many digital systems. Various truncation error compensation (TEC) schemes have been presented for FWBM designs, aiming to reduce hardware costs while preserving operation accuracy. In general, the existing TEC methods function adequately for an exact bit width of the operand but fail to consider the TEC effect for FWBM inputs with various bit-width levels. To address this issue, we propose a bit-width adaptive TEC (BWATEC) scheme for providing high-accuracy TEC functions that are adaptive to the multiple $L^{\prime}$-bit numerical ranges of input data for an $L$-bit FWBM $\left(L^{\prime} \leq L\right)$. We also present adjustable architecture for a 16-bit FWBM to enable the proposed BWATEC scheme and evaluate the hardware performance, using the TSMC $40 \mathrm{~nm}$ standard cell library. Relative to the contrast 16-bit FWBM approaches that use state-of-the-art TEC methods, the proposed BWATEC-enabled FWBM design can achieve reductions in the area-delay-error product of 7.9-50.9\%, 17.1-69.5\%, 29.9-82.2\%, and 100\% for the 14-bit, 12-bit, 10-bit, and 8-bit inputs, respectively. Moreover, the resultant 16-bit FWBM with BWATEC was verified by using the field-programmable gate array for convolutional neural network acceleration.
\end{abstract}

Keywords: Booth multiplier; fixed-width; truncation error compensation; bit-width adaptive

\section{Introduction}

Multipliers are widely used in many digital operation systems. To limit bit-width increases in data paths, fixed-width multipliers are accordingly employed as arithmetic modules for digital signal processing, communication baseband operations, and neural network acceleration [1-4]. L-bit fixed-width multipliers generate the same L-bit output width as the L-bit operand, of which the Baugh-Wooley (array) multiplier and Booth multiplier are two of the most popular types. Two convenient approaches to fixed-width Baugh-Wooley or Booth multipliers are post-truncation (PT) and direct-truncation (DT). The PT method calculates all partial products and rounds the $2 L$-bit full-width product to the $L$ most significant bits (MSBs) to achieve high accuracy, but the hardware costs are high. The DT method truncates the partial products related to the least significant bits (LSBs) of the $2 L$-bit full-width product to reduce the hardware costs, but the accuracy is very low.

Considering both operation accuracy and hardware complexity, several schemes based on truncation error compensation (TEC) have been presented for fixed-width BaughWooley multipliers [5-8] or fixed-width Booth multipliers (FWBMs) [9-22]. The Booth multiplier has benefits in achieving high hardware efficiency because the number of rows of partial products is significantly reduced [23,24]. Moreover, the lower level of truncated partial products for Booth multipliers profits the fixed-width operation accuracy $[17,18]$. Therefore, the FWBM that enables a kind of TEC scheme is discussed in this study. A number of TEC schemes for FWBMs have been presented [9-22]. In general, TEC schemes for FWBMs obtain a TEC value (bias) based on computer simulation [9-14] or probability-based estimation [14-22] to compensate for the truncation error associated with the curtailed 
partial products. For simulation-based methods, the work in Reference [9] used linear regression analysis and simulation to generate bias values. In Reference [10], bias terms were generated based on simulation outcomes, and simplified through the Karnaugh map processing. Moreover, the authors of References [11,12] derived formulas in a closed form for TEC biasing based on simulation results. In References [12-14], the bias terms through a simulation were determined utilizing the Booth encoded results to further improve accuracy. The computer simulation methods presented in References [9-14] are applicable but generally consume exhaustive simulation time to obtain the TEC bias value. Instead of exhaustive simulation, the authors of References [14-22] also presented the probability-based scheme to derive the TEC function. In this study, we aim to design the TEC-enabled FWBM based on the probabilistic estimation method. More literature reviews for the state-of-the-art FWBM design that uses the probability-based TEC scheme [14-22] are discussed in Section 2.2.

In conventional TEC methods for FWBMs, TEC functions are generally operated based on a certain particular bit width of the FWBM operand. However, in practical applications, an $L$-bit FWBM might need to process input patterns with various $L^{\prime}$-bit widths $\left(L^{\prime} \leq L\right.$; $L$ and $L^{\prime}$ are generally even). For example, a 16-bit FWBM might be employed to operate with 16-bit, 14-bit, 12-bit, 10-bit, or 8-bit numerical input patterns, as specified by different situations. Such an operation can be practically performed in several applications. Taking the convolutional neural network $(\mathrm{CNN})$ as an example, an accelerator may employ an FWBM to process input data that have different settable bit widths from different CNN models or layers. Moreover, FWBMs used in a shared digital filter might operate with input data whose levels are various for multiple analog modules. To the best of our knowledge, no previous study has developed a TEC scheme for such an FWBM design to offer adaptive TEC biasing for various bit widths of input data. In this study, we propose a bit-width adaptive TEC (BWATEC) scheme for providing an adjustable TEC bias for the diverse bit widths of input patterns. For an $L$-bit FWBM, the proposed BWATEC method can enable a tailored and high-accuracy TEC function for each case of the $L^{\prime}$-bit input pattern (where $L^{\prime} \leq L$ ). In addition, an FWBM design for enabling the BWATEC is proposed based on a reconfigurable bias circuit with high hardware efficiency.

The remainder of this paper is organized as follows. Section 2 briefly introduces the background of FWBMs and the conventional probability-based TEC schemes for FWBMs. Section 3 outlines the proposed BWATEC scheme and its operations. In Section 4, the architecture of a 16-bit FWBM enabling the proposed BWATEC scheme is described. Section 5 evaluates the accuracy and hardware performances of our design and reports the experiment results, using a system-on-chip (SoC) field-programmable gate array (FPGA) platform. Finally, the conclusions are highlighted in Section 6.

\section{Preliminaries and Design Issues}

Some abbreviations and acronym words frequently used in this study are tabulated in Table 1 for convenient reference.

Table 1. Mapping of abbreviations and acronym words.

\begin{tabular}{cc}
\hline FWBM & Fixed-Width Booth Multiplier \\
\hline TEC & Truncation error compensation \\
\hline BWATEC & Bit-width adaptive truncation error compensation \\
\hline P.P. & Partial products \\
\hline MP/TP & Main part/truncation part \\
\hline MSC & Convolutional neural network \\
\hline CNN & Electrocardiogram \\
\hline ECG &
\end{tabular}


Table 1. Cont.

\begin{tabular}{cc}
\hline FWBM & Fixed-Width Booth Multiplier \\
\hline HW/SW & Hardware/software \\
\hline SoC-FPGA & System-on-chip field-programmable gate array \\
\hline ZP & Zero-Padding \\
\hline $\mathrm{R}_{\mathrm{Z}} / \mathrm{R}_{\mathrm{H}} / \mathrm{R}_{\mathrm{D}}$ & Zero region/hybrid region/deterministic-only region \\
\hline $\mathrm{ADEP}$ & Area-delay-error product \\
\hline
\end{tabular}

\subsection{Fixed-Width Booth Multiplier (FWBM)}

Let $A$ and $B$ be two $L$-bit 2's complement operands, represented by " $a_{L-1}, a_{L}, \ldots, a_{1}$, $a_{0}$ " and " $b_{L-1}, b_{L}, \ldots, b_{1}, b_{0}$ " with the values shown below, respectively.

$$
A=-a_{L-1} \cdot 2^{L-1}+\sum_{i=0}^{L-2} a_{i} \cdot 2^{i} \quad B=-b_{L-1} \cdot 2^{L-1}+\sum_{i=0}^{L-2} b_{i} \cdot 2^{i}
$$

The Booth encoding maps three consecutive terms, $b_{2 j+1}, b_{2 j}$, and $b_{2 j-1}$ into $d_{j}$, as tabulated in Table 2 . The $d_{j}$ value can be associated with $\left(b_{2 j+1}, b_{2 j}, b_{2 j-1}\right)$ terms as expressed in Equation (2), where $Q=(1 / 2) \times L$. As a result, a $2 L$-bit full-width product $(F P)$ for $A \times B$ can be obtained as shown in Equation (3).

$$
\begin{gathered}
B=\sum_{j=0}^{Q-1} d_{j} \cdot 2^{2 j}, d_{j}=-2 b_{2 j+1}+b_{2 j}+b_{2 j-1} \\
F P=\left(\sum_{j=0}^{Q-1} d_{j} \cdot 2^{2 j}\right) \times\left(-a_{L-1} \cdot 2^{L-1}+\sum_{i=0}^{L-2} a_{i} \cdot 2^{i}\right)
\end{gathered}
$$

\begin{tabular}{|c|c|c|c|}
\hline$\left(\begin{array}{lll}b_{2 j+1} & b_{2 j} & b_{2 j-1}\end{array}\right)$ & $d_{j}$ & $p_{L, j} p_{L-1, j} p_{L-2, j}$ & $n_{j}$ \\
\hline$\left(\begin{array}{lll}0 & 0 & 0\end{array}\right) /\left(\begin{array}{llll}1 & 1 & 1\end{array}\right)$ & 0 & $\begin{array}{lll}0 & \cdots & 0\end{array}$ & 0 \\
\hline$\left(\begin{array}{llll}0 & 0 & 1\end{array}\right) /\left(\begin{array}{lllll}0 & 1 & 0\end{array}\right)$ & 1 & $\begin{array}{ccccccc}a_{L-1} & a_{L-1} & a_{L-2} & \cdots & a_{2} & a_{1} & a_{0}\end{array}$ & 0 \\
\hline$\left(\begin{array}{lll}1 & 0 & 1\end{array}\right) /\left(\begin{array}{lll}1 & 1 & 0\end{array}\right)$ & -1 & 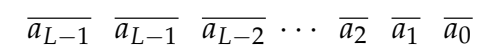 & 1 \\
\hline$\left(\begin{array}{lll}0 & 1 & 1\end{array}\right)$ & 2 & $\begin{array}{cccccc}a_{L-1} & a_{L-2} & a_{L-3} & \cdots & a_{1} & a_{0} \\
\end{array}$ & 0 \\
\hline$\left(\begin{array}{lll}1 & 0 & 0\end{array}\right)$ & -2 & $\overline{a_{L-1}} \overline{a_{L-2}} \quad \overline{a_{L-3}} \cdots \overline{a_{1}}$ & 1 \\
\hline
\end{tabular}

Table 2. Mapping results for the Booth encoder and partial products.

Using binary arithmetic for $A \times B$, the partial products (P.P.) for each $d_{j}$ can be derived in terms of $a_{i}(i$ is from 0 to $L-1), 0$, or 1 , as shown for the values of $p_{i, j}$, and $n_{j}$ in Table 2 . Based on the P.P. terms in Table 2, Figure 1 depicts the structure of the P.P. array for an example of a 16-bit $(L=16) A \times B$ full-width Booth multiplier. As shown in Figure 1, all $P . P$. terms can be divided into two groups: the main part $(M P)$ and truncation part $(T P)$. The P.P. in the MP are calculated to generate the product, whereas the TP includes the $P . P$. for computing the rounded $L$ LSBs of the full-width product. The TP can be further divided into the $T P_{\text {major }}$ and $T P_{\text {minor }}$ subgroups. As indicated in Figure 1, $T P_{\text {major }}$ contains the P.P. in the most significant column (MSC) of the TP, which dominates the accuracy of the carry from the TP toward the MP. In general, the accuracy can be improved by increasing the column range for $T P_{\text {major }}$ [17-20]. However, a TEC function based on the MSC TP $P_{\text {major }}$ with one MSC usually offers adequate accuracy in many applications and the use of one-MSC $T P_{\text {major }}$ sufficiently serves as a baseline to evaluate the performances for different TEC schemes $[12,14,16,22]$. Thus, this study adopted the one-MSC TP major to develop and evaluate our BWATEC scheme and FWBM design. In an FWBM design with 
TEC, $T P_{\text {major }}$ is reserved for calculation, whereas $T P_{\text {minor }}$ is truncated, and an estimated bias is adopted to compensate for the truncation error [9-22]. Therefore, an L-bit FWBM with TEC produces an $L$-bit quantized $F P_{q}$ result, as expressed in Equation (4), where $B_{T E C}$ indicates the estimated bias value for TEC, $T P_{\text {major }}$ is mapped to the $2^{-1}$ digit and $R\{$.$\} is$ the rounding operation.

$$
F P_{q}=M P+\sigma \cdot 2^{L}, \sigma=R\left\{T P_{\text {major }}+B_{T E C}\right\}
$$

With regard to an $L$-bit FWBM whose operands can be assigned to the input data of multiple prespecified $L^{\prime}$-bit width $\left(L^{\prime} \leq L\right)$, the $L^{\prime}$-bit input patterns are necessarily left-shifted by $\left(L-L^{\prime}\right)$ bits and are padded with zeros (i.e., Zero-Padding bits) to form the $L$-bit operand. The aforementioned processing for $L=16$ is also described in Figure 1 for input patterns with multiple 14-bit, 12-bit, 10-bit, or 8-bit (i.e., $L^{\prime}$-bit) widths.

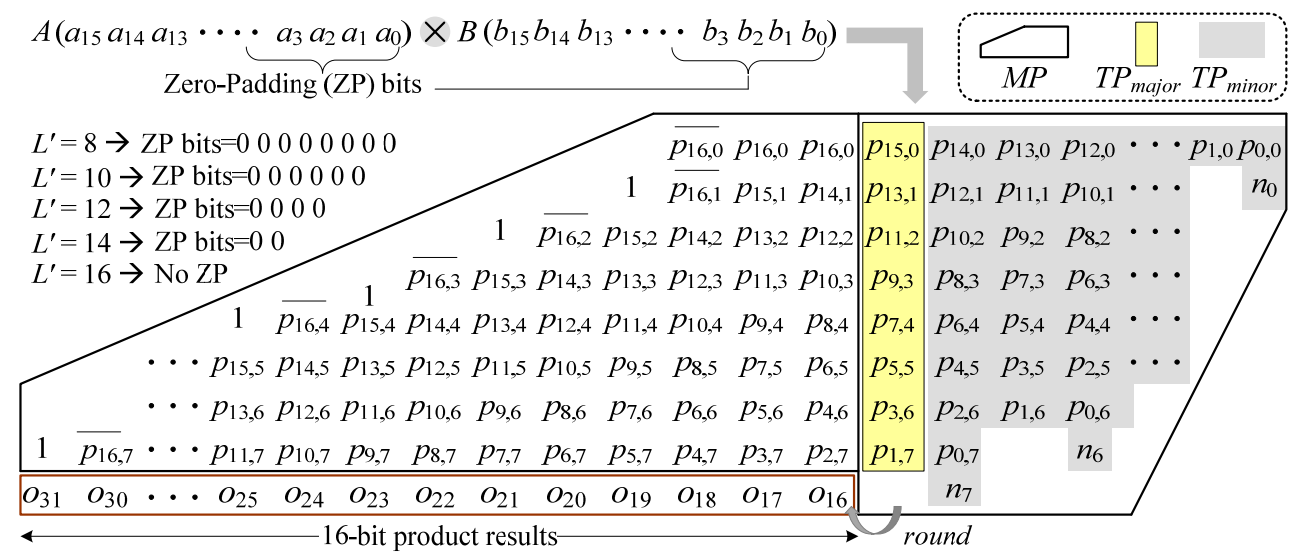

Figure 1. Partial product (P.P.) array structure for a 16-bit full-width Booth multiplier with multiple 16-to-8-bit numerical ranges of input patterns.

\subsection{Probability-Based TEC Schemes for FWBMs}

Several FWBM designs with probability-based TEC have been presented [14-22]. The authors of Reference [14] presented the probability-based scheme, together with their simulation-based works. Similarly, the work in Reference [15] used the expected value for P.P. to derive bias values. Furthermore, the probabilistic analysis methods [16-22] derived closed formulas of the TEC function based on the expected value or the conditional probability for P.P. terms. In Reference [16], the expected values for two groups of $T P_{\text {minor }}$ (i.e., the $n_{j}$ terms in Table 2 equals to 0 or 1 ) were respectively derived to obtain the probabilistic estimation bias when one-MSC $T P_{\text {major }}$ is specified. In addition, a generalized probabilistic estimation bias (GPEB) method [17] further enhanced the work in Reference [16] for the cases of $T P_{\text {major }}$ containing more P.P. columns. Using the GPEB methods $[16,17]$, a simple TEC function of a 1-bit or 2-bit constant value was derived. The work in Reference [18] presented a TEC scheme based on the conditional probability depending on non-zero Booth encoder outputs (i.e., $d_{j} !=0$ in Table 2 ) for each row of $T P_{\text {minor }}$. In Reference [19], a more complex method based on [18] was presented by using a conditional probability model for multiple $T P_{\text {minor }}$ rows. Such a design [19] slightly improved accuracy but increased hardware overheads. The authors of Reference [20] considered both expected values and conditional probability to progress a bias function improving accuracy and area based on the probability and computer simulation (PACS). In Reference [21], the concept of data scaling was presented and applied to conventional TEC-adapted FWBM designs for improving accuracy. A Booth-encoded sign-digit-based conditional probability (BSCP) method was presented in Reference [22] for the case of one-MSC TP major. The work in Reference [22] further took advantage of the sign of non-zero Booth encoder results to generate a TEC function achieving relatively high accuracy. 
Considering a 16-bit FWBM design with TEC, the aforementioned conventional TEC schemes can be directly applied to the design example, as shown in Figure 1 . However, such approaches cannot achieve optimized accuracy for input patterns with 14/12/10/8-bit widths, as the applied TEC functions are for 16-bit operands; thus, imprecise biasing might be introduced to 14-bit to 8-bit FWBM operations. Accordingly, the development of an enhanced and tailored TEC scheme (e.g., the proposed BWATEC method) that is adaptive to input patterns with values in multiple bit-width levels is considered to be useful and practical for the TEC-enabled FWBM design.

\section{Proposed Bit-Width Adaptive TEC (BWATEC) Scheme}

In Sections 3.1 and 3.2, we use the 16-bit FWBM as an example for explaining the probability-based bias estimation and TEC operations for the proposed BWATEC scheme.

\subsection{Derivation of Probabilistic Estimation for BWATEC}

Referring to Figure 1 , there are eight rows of $T P_{\text {minor }}$ (incl. $n_{j}$ ) in the P.P. array for the 16-bit $A \times B$ Booth multiplication. We can represent a row index of $j$ from 0 to 7 (the top row is the 0 th row). The contents of the TP vary with the number of Zero-Padding (ZP) bits for different $L^{\prime}$-bit input patterns of the operands $A$ and $B$. Based on the mapping results from Table 2, Figure 2a-c illustrates the contents of $T P_{\text {minor }}$ for $L^{\prime}=14,12$, and 10, respectively. As described in Figure $2 \mathrm{a}-\mathrm{c}, T P_{\text {minor }}$ is classified into three regions: the zero region $\left(R_{Z}\right)$, hybrid region $\left(R_{H}\right)$, and deterministic-only region $\left(R_{D}\right)$. The $R_{Z}$ region only has zero-valued P.P. related to the Booth-encoded result of the ZP bits for the $B$ operand, and thus can be trivially truncated. In Figure 2, the $\mathrm{R}_{\mathrm{H}}$ region includes P.P. with hybrid deterministic and probabilistic values. For the $j$ th row of $T P_{\text {minor }}$ in $\mathrm{R}_{\mathrm{H}}$, the $s_{j}$ terms are the P.P. associated with the ZP bits of the operand $(A)$. Both $n_{j}$ and $s_{j}$ can be exactly determined to be " 0 " or " 1 " (i.e., deterministic values) depending on $d_{j}$, based on the contents in Table 2 . The $e_{j}$ in $\mathrm{R}_{\mathrm{H}}$ (Figure 2) is the P.P. value of the $p_{r, j}$ (wherein $r=L-L^{\prime}$ ). From Table 2, it can be observed that the $e_{j}$ value can be equal to " 0 " or " 1 " $\left(d_{j}= \pm 2\right)$ or can be identified by the LSB of the original $L^{\prime}$-bit input data $\left(d_{j}= \pm 1\right)$. In the $\mathrm{R}_{\mathrm{H}}$ region, the $e_{j}$ terms in the case of $\left(d_{j}= \pm 1\right)$ and all other P.P. terms, excluding $n_{j}, \mathrm{~s}_{j}$, and $e_{j}$, can be estimated by using an expected value of $1 / 2$ (i.e., probabilistic values) [16-20]. Relative to the $\mathrm{R}_{\mathrm{H}}$, all P.P. in the $\mathrm{R}_{\mathrm{D}}$ region are only $s_{j}$ and $n_{j}$ terms, which are deterministic values. In addition to the cases of $L^{\prime}=14,12$, and 10 (shown in Figure $2 \mathrm{a}-\mathrm{c}$ ), the $T P_{\text {minor }}$ contents for two contrast cases of $L^{\prime}=16$ and $L^{\prime}=8$ are also illustrated in Figure $3 \mathrm{a}, \mathrm{b}$. For $L^{\prime}=16, T P_{\text {minor }}$ only has the $\mathrm{R}_{\mathrm{H}}$ region, while when $L^{\prime}=8$, only the $R_{Z}$ and $R_{D}$ regions are included.

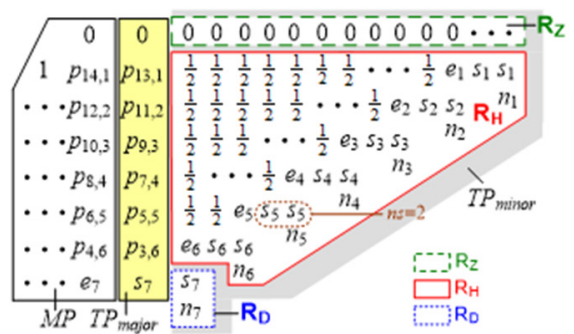

(a)

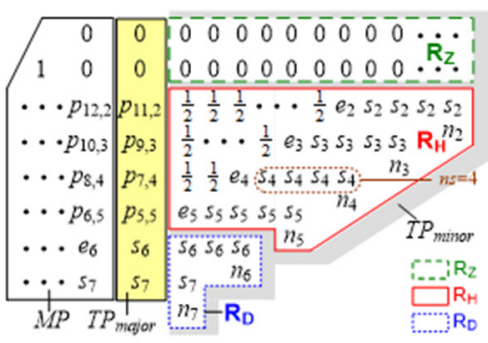

(b)

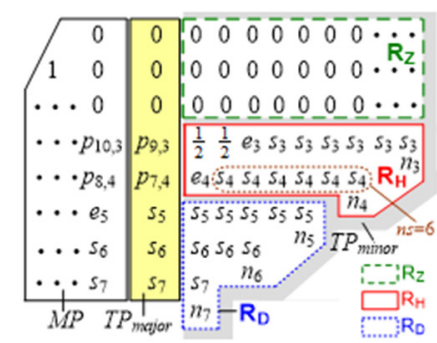

(c)

Figure 2. Schematic for the $T P_{\text {minor }}$ contents in a P.P. array of a 16-bit Booth multiplier for various $L^{\prime}$-bit input patterns: (a) $L^{\prime}=14$, (b) $L^{\prime}=12$, and (c) $L^{\prime}=10$. 


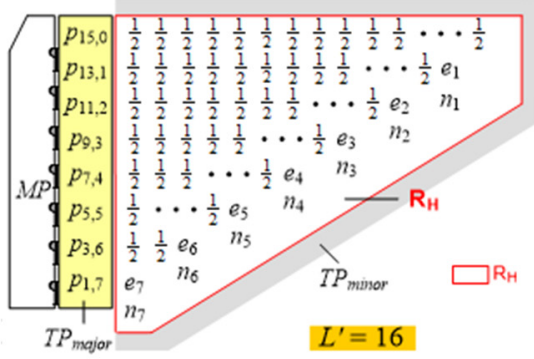

(a)

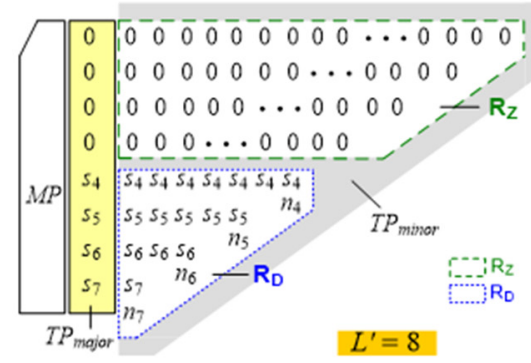

(b)

Figure 3. Schematic for the $T P_{\text {minor }}$ contents of a 16-bit Booth multiplier for other $L^{\prime}$-bit inputs: (a) $L^{\prime}=16$ and (b) $L^{\prime}=8$.

By mapping $T P_{\text {major }}$ to the $2^{-1}$ digit (i.e., the MSB of $T P_{\text {minor }}$ is $2^{-2}$ ), the expected value of all P.P. for the $j$ th-row $T P_{\text {minor }}$ in the $\mathrm{R}_{\mathrm{H}}, E\left[T P_{\text {minor, }}^{(\mathrm{H})}\right]$, can be calculated as Equation (5), where $n s$ is the number of $s_{j}$ (refer to Figure 2). Based on Equation (5) and the mapping contents in Table 2 , the values of $E\left[T P_{\text {minor, }}^{(\mathrm{H})}\right]$ and $\left(n_{j}, \mathrm{~s}_{j}, e_{j}\right)$ according to $d_{j}$ are listed in Table 3.

$$
2^{2 j-16} \cdot n_{j}+\sum_{\alpha=0}^{n s-1} 2^{2 j+\alpha-16} \cdot s_{j}+2^{2 j+n s-16} \cdot e_{j}+\sum_{\beta=n s+1}^{14-2 j} 2^{2 j+\beta-16} \cdot 1 / 2
$$

Table 3. Values of $\left(n_{j}, \mathrm{~s}_{j}, e_{j}\right)$ and according to $d_{j}$.

\begin{tabular}{ccc}
\hline & $P . P$. Values & $\boldsymbol{E}\left[\mathbf{T P}_{\text {minor }, j}^{(\mathbf{H})}\right]$ Values \\
\hline$d_{j}=0$ & $($ all $P . P .=0)$ & zero \\
\hline$d_{j}=1$ & $\left(n_{j}=0 ; s_{j}=0 ; e_{j}=1 / 2\right)$ & $2^{-2}-2^{-16+2 j+n s-1}$ \\
\hline$d_{j}=-1$ & $\left(n_{j}=1 ; s_{j}=1 ; e_{j}=1 / 2\right)$ & $2^{-2}+2^{-16+2 j+n s-1}$ \\
\hline$d_{j}=2$ & $\left(n_{j}=0 ; s_{j}=0 ; e_{j}=0\right)$ & $2^{-2}-2^{-16+2 j+n s}$ \\
\hline$d_{j}=-2$ & $\left(n_{j}=1 ; s_{j}=1 ; e_{j}=1\right)$ & $2^{-2}+2^{-16+2 j+n s}$ \\
\hline
\end{tabular}

The $E\left[T P_{\text {minor, }}^{(\mathrm{H})}\right]$ values in Table 3 can be summarized by using the following expression, where a variable $\delta_{j}$ is defined by $\delta_{j}=1$ for $d_{j} !=0$; otherwise, $\delta_{j}=0$.

$$
E\left[T P_{\text {minor }, j}^{(\mathrm{H})}\right]=2^{-2} \cdot \delta_{j}-d_{j} \cdot 2^{-16+2 j+n s-1}
$$

From observing the $\mathrm{R}_{\mathrm{H}}$ region for $L^{\prime}=14,12$, and 10 in Figure 2, it can be found that the $\mathrm{R}_{\mathrm{H}}$ includes the $m$ th to the $k$ th row of $T P_{\text {minor, }}$, in which $m=n s / 2$ and $k=7-(n s / 2)$. By summing $E\left[T P_{\text {minor, },}^{(\mathrm{H})}\right]$ for all rows in the $\mathrm{R}_{\mathrm{H}}$, an overall $E\left[T P_{\text {minor }}^{(\mathrm{H})}\right]$ can be obtained as follows:

$$
E\left[T P_{\text {minor }}^{(\mathrm{H})}\right]=\sum_{j=m}^{k} E\left[T P_{\text {minor }, j}^{(\mathrm{H})}\right]=\sum_{j=m}^{k} 2^{-2} \cdot \delta_{j}-\sum_{j=m}^{k} d_{j} \cdot 2^{-16+2 j+n s-1}
$$

For an FWBM with TEC, the result of Equation (7) can be viewed as an ideal bias for the truncated $T P_{\text {minor }}$ in the $\mathrm{R}_{\mathrm{H}}$; however, the calculation of (a) in Equation (7) is complex. The bottom row in the $\mathrm{R}_{\mathrm{H}}$ (i.e., the $k$ th row; $j=k$ ) dominates the final calculation result. Moreover, the result of (a) in Equation (7) can be rounded to the $2^{-2}$ digit to be arithmetically added to $\delta_{j}$. Therefore, Equation (7) can be approximated by Equation (8) by 
simplifying the (a) part to a $\sigma \cdot 2^{-2}$ term, where $R_{-2}\{$.$\} represents rounding a value to the$ $2^{-2}$ digit.

$$
\begin{gathered}
E\left[T P_{\text {minor }}^{(\mathrm{H})}\right] \cong \sum_{j=m}^{k}\left(2^{-2} \cdot \delta_{j}\right)-R_{-2}\left\{d_{k} \cdot 2^{-3}\right\} \cong \sum_{j=m}^{k}\left(2^{-2} \cdot \delta_{j}\right)+\sigma \cdot 2^{-2} \\
, \sigma=1, d_{k}<0 ; \sigma=-1, d_{k}>0 ; \sigma=0, d_{k}=0
\end{gathered}
$$

However, the subtraction arithmetic for the $2^{-2}$ (i.e., $\sigma=-1$ in Equation (8)) is also an issue in a P.P. array. This issue can be resolved by taking advantage of the following operational features. When $d_{k}$ is negative, both $\delta_{k}$ and $\sigma$ are equal to 1 ; thus, a carry of " 1 " can be added to the $2^{-1}$ digit. If $d_{k}$ is positive, $\delta_{k}$ is 1 , whereas $\sigma$ is -1 . Thus, $\delta_{k}$ can be eliminated at the $2^{-2}$ digit, owing to the offset by $\sigma$. As a result, Equation (8) can be further calculated by using Equation (9), where a variable, $\gamma$, is operated at the $2^{-1}$ digit only with an addition.

$$
E\left[T P_{\text {minor }}^{(\mathrm{H})}\right] \cong \sum_{j=m}^{k-1}\left(2^{-2} \cdot \delta_{j}\right)+2^{-1} \cdot \gamma ; \gamma=\left\{\begin{array}{l}
1, d_{k}<0 \\
0, d_{k} \geq 0
\end{array}\right.
$$

As shown in Figure $2 \mathrm{a}-\mathrm{c}$, the $\mathrm{R}_{\mathrm{D}}$ region only comprises P.P. in terms of $s_{j}$ and $n_{j}$, as the number of P.P. within a row in the $R_{D}$ is less than the number of ZP bits (Figure 1). Similar to $s_{j}$ in the $\mathrm{R}_{\mathrm{H}}$, the $s_{j}$ terms in the $\mathrm{R}_{\mathrm{D}}$ are also P.P. obtained from the ZP bits of the $A$ operand and are equal to the $d_{j}$-dependent deterministic " 1 " or " 0 " values. Setting $s_{j}$ and $n_{j}$ to " 1 " (for $d_{j}<0$ ) or " 0 " (for $d_{j} \geq 0$ ), the actual value of all P.P. for the $j$ th row of $T P_{\text {minor }}$ in the $\mathrm{R}_{\mathrm{D}}$, i.e., $E\left[T P_{\text {minor, }}^{(\mathrm{D})}\right]$, can be obtained by the following derivation. An accumulated result from the $j$ th row in the $R_{D}$ is introduced to the $2^{-1}$ digit for negative $d_{j}$ values.

$$
E\left[T P_{\text {minor }, j}^{(\mathrm{D})}\right]=2^{-16+2 j} \cdot\left(n_{j}+s_{j}\right)+\cdots+2^{-2} \cdot s_{j}=\left\{\begin{array}{c}
0, d_{j} \geq 0 \\
2^{-1}, d_{j}<0
\end{array}\right.
$$

For the design examples illustrated in Figure $2 \mathrm{a}-\mathrm{c}$, the $\mathrm{R}_{\mathrm{D}}$ region includes rows with indexes from $k+1$ to $Q-1$, where $Q$ equals 8 for the case of $L=16$. The variable $Q$ is defined as $Q=L / 2$, which is the number of rows in a P.P. array. Thus, a global $E\left[T P_{\text {minor }}^{(\mathrm{D})}\right]$ can be derived, as shown in Equation (11), in which the variable $\lambda_{j}$ is defined by $\lambda_{j}=1$ for $d_{j}<0$ and $\lambda_{j}=0$ for $d_{j} \geq 0$, corresponding to the execution results of Equation (10) for each row.

$$
E\left[T P_{\text {minor }}^{(\mathrm{D})}\right]=\sum_{j=k+1}^{Q-1} T P_{\text {minor }, j}^{(\mathrm{D})}=\sum_{j=k+1}^{Q-1}\left(2^{-1} \cdot \lambda_{j}\right)
$$

\subsection{BWATEC Synthesis and Operations}

For FWBMs with TEC, $E\left[T P_{\text {minor }}^{(\mathrm{H})}\right]$ and $E\left[T P_{\text {minor }}^{(\mathrm{D})}\right]$ values obtained by using Equations (9) and (11) can be employed as the TEC bias to compensate for the truncated P.P. of TP minor in the $R_{H}$ and $R_{D}$, respectively. Moreover, the operations of Equations (9) and (11) are different from the input bit width $\left(L^{\prime}\right)$, as well as the contents and range of the $R_{H}$ and $R_{D}$ regions (Figure 2). In addition to the cases of $L^{\prime}=14,12$, and 10 as shown in Figure 2a-C, the proposed schemes based on Equations (9) and (11) can also be applied to the conditions of $L^{\prime}=16$ and 8 as shown in Figure 3 . For $L^{\prime}=16, T P_{\text {minor }}$ only has the $\mathrm{R}_{\mathrm{H}}$ region, and we can use Equation (9) to generate the TEC bias. Alternatively, when $L^{\prime}=8$, Equation (11) is used, as only the $\mathrm{R}_{\mathrm{D}}$ region is calculated. In practice, the TEC function for $L^{\prime}=16$ can be further improved. From Figure 1, we can use deterministic $p_{0,7}$ and $n_{7}$ to operate with $\delta_{j}$ at the $2^{-2}$ digit; thus, a more precise carry can be added at the $2^{-1}$ digit, instead of adding $\gamma$ in Equation (9). The efficient use of Equations (9) and (11), as associated with multiple combinations of deterministic and probabilistic data, achieves the aims of the proposed BWATEC scheme. Considering a 16-bit FWBM, Figure 4 illustrates the TEC operations by using the proposed BWATEC scheme for various $L^{\prime}$-bit inputs. 

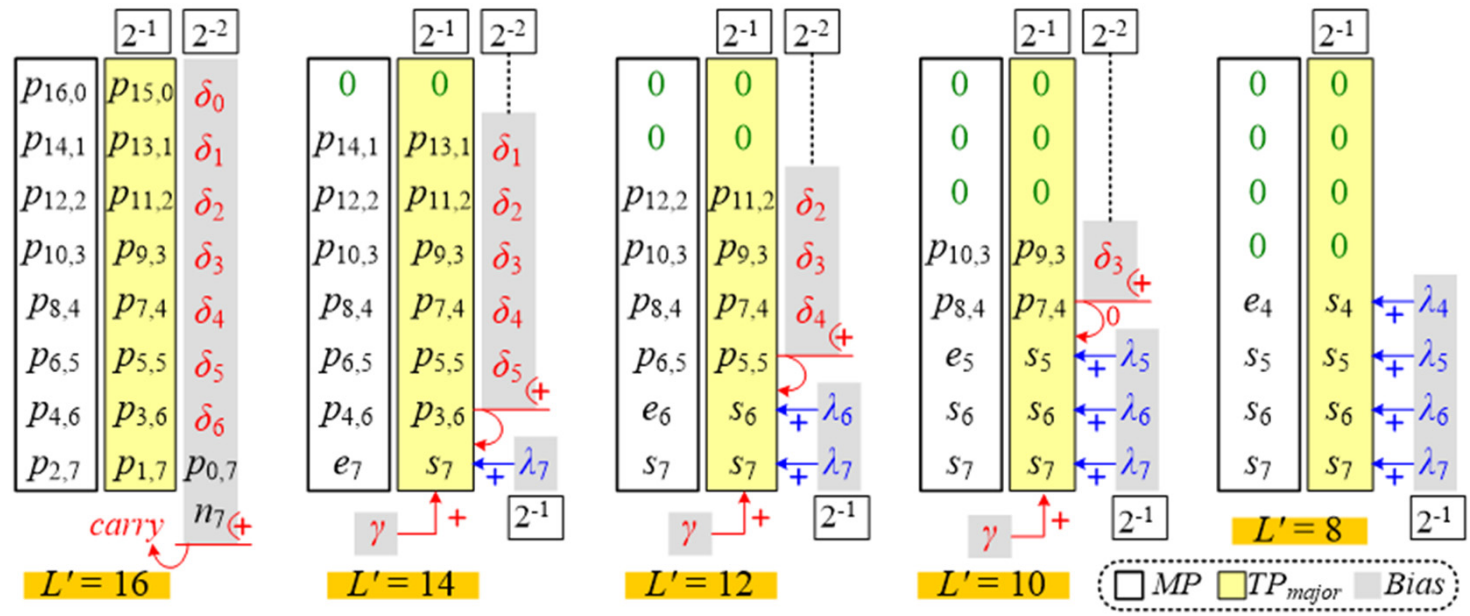

Figure 4. Schematic of truncation error compensation (TEC) operations that use the proposed bit-width adaptive TEC (BWATEC) scheme for various $L^{\prime}$-bit input patterns of a 16-bit fixed-width Booth multiplier (FWBM).

\subsection{Design Scalability}

Taking the 16-bit FWBM example as a base, the deduced processing can also be applied to general $L$-bit FWBM designs (i.e., $L$ is a scalable number other than 16). In general, an $L$ bit Booth multiplier is operated based on $L$ of an even number. Considering the scalability of the proposed design, the aimed L-bit FWBMs can be categorized into two kinds of specifications. One is $L=2 n$, and $n$ is an even integer; thus, the number of P.P. rows (i.e., the $Q$ value) is even based on $Q=L / 2$. The other is $L=2 n$, and $n$ is an odd integer; thus, the number of P.P. rows is odd. Referring to the contents associated with Figures 2 and 3 , the $T P_{\text {minor }}$ P.P. corresponding to the $\mathrm{R}_{\mathrm{Z}} / \mathrm{R}_{\mathrm{H}} / \mathrm{R}_{\mathrm{D}}$ regions are illustrated for the design case of an $L$-bit Booth multiplier $(L=16)$ with various $L^{\prime}$-bit inputs $\left(L^{\prime}=16,14,12,10\right.$, and 8$)$, which has even (i.e., 8) P.P. rows. Moreover, the contents related to Figure 4 illustrate the proposed BWATEC operation for a 16-bit FWBM. As extension based on the illustration for the case of $L=16$, Figure 5 depicts the $R_{Z} / R_{H} / R_{D}$ distribution of $T P_{\text {minor }}$ rows of a general $L$-bit Booth multiplier (i.e., $L$ is scalable) for different $L^{\prime}$-bit inputs, and Figure $5 \mathrm{a}, \mathrm{b}$ illustrates the specification of " $L=2 n$ ( $n$ and $Q$ are even; even rows)" and " $L=2 n$ ( $n$ and $Q$ are odd; odd rows)", respectively. In Figure $5 a$,b, the value shown inside each $R_{Z} / R_{H} / R_{D}$ block represents the number of rows in that region and refers to the ceiling operator.

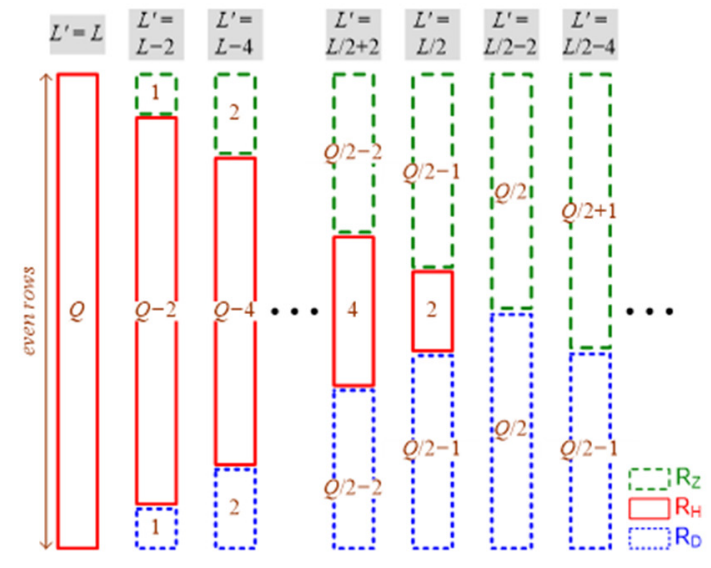

(a)

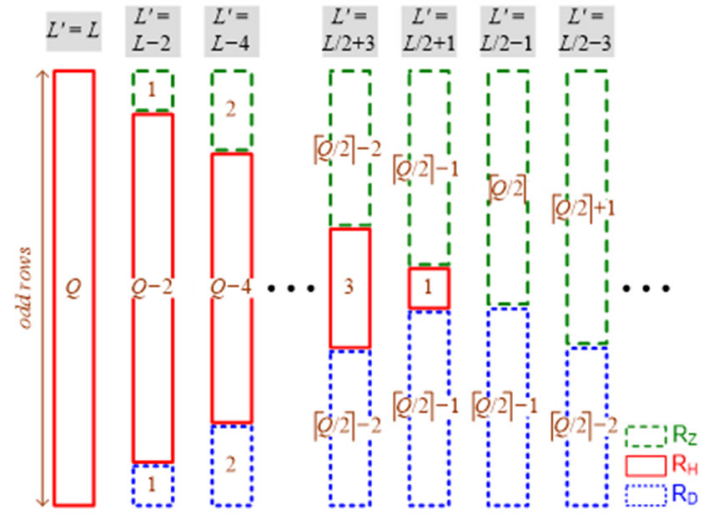

(b)

Figure 5. Schematic for the $\mathrm{R}_{\mathrm{Z}} / \mathrm{R}_{\mathrm{H}} / \mathrm{R}_{\mathrm{D}}$ distribution of $T P_{\text {minor }}$ rows of a general $L$-bit Booth multiplier for various $L^{\prime}$-bit inputs: (a) $L=2 n$, and $n$ is even; (b) $L=2 n$, and $n$ is odd. 
As indicated in the previous section, the proposed BWATEC operations for the case of a 16-bit FWBM (i.e., Figure 4) can be synthesized based on the contents in Figures 2 and 3 in common with Equations (9) and (11). By analogy with the derivation for the contents in Figure 4, the proposed BWATEC operations for various $L^{\prime}$-bit input patterns of a general L-bit FWBM can be similarly synthesized based on Figure 5, Equations (9) and (11), as described in Figure 6, where the two specifications of $L=2 n$ ( $n$ is even or odd) are also respectively illustrated. The contents in Figures 5 and 6 further address the $R_{Z} / R_{H} / R_{D}$ distribution and BWATEC operations of a general $L$-bit FWBM operated with $L^{\prime}$-bit inputs in small $L^{\prime}$ values. For the cases of " $L^{\prime} \leq L / 2-2\left(L=2 n ; n\right.$ is even)" or " $L^{\prime} \leq L / 2-1(L=2 n$; $n$ is odd)", only the $R_{Z}$ and $R_{D}$ regions are included and the range of $R_{D}$ is reduced with smaller $L^{\prime}$ values. Such conditions allow only the $R_{D}$ P.P. to be calculated to obtain the TEC bias, as expressed in Equation (12), which is an extended form based on Equation (11).

$$
E\left[T P_{\text {minor }}^{(\mathrm{D})}\right]=\sum_{j=t}^{Q-1}\left(2^{-1} \cdot \lambda_{j}\right) ; t=\left\{\begin{array}{l}
Q / 2+1, L^{\prime} \leq L / 2-2, \text { Qiseven } \\
\lceil Q / 2\rceil+1, L^{\prime} \leq L / 2-1, \text { Qisodd }
\end{array}\right.
$$

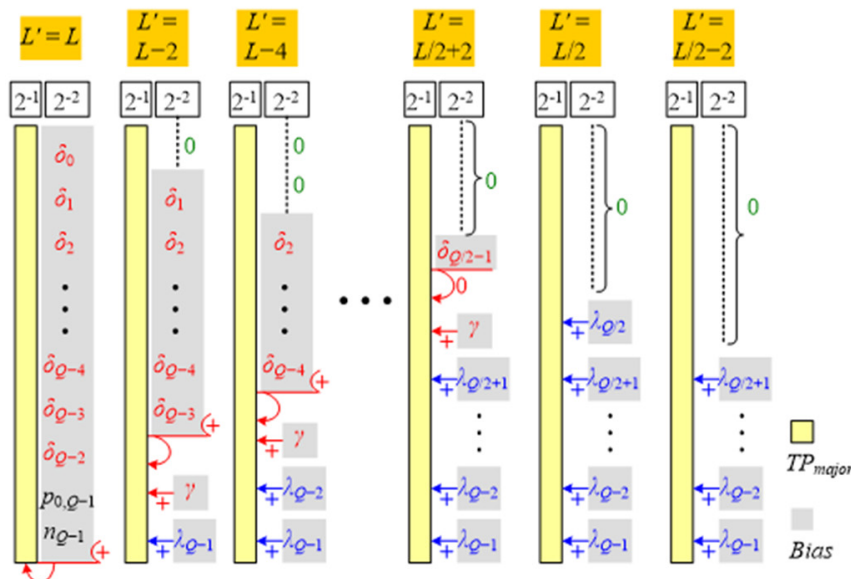

(a)

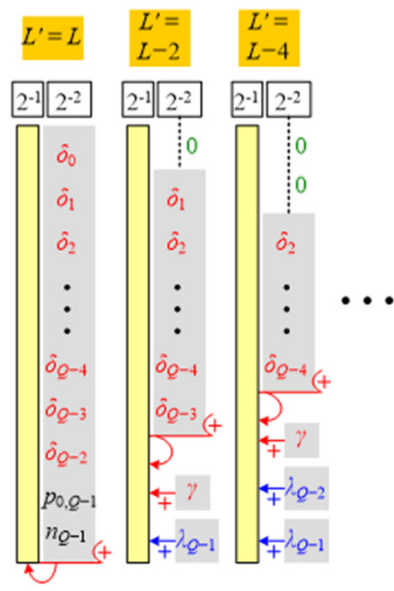

(b)

Figure 6. Schematic of BWATEC operations for various $L^{\prime}$-bit input patterns of a general $L$-bit FWBM: (a) $L=2 n$, and $n$ is even; (b) $L=2 n$, and $n$ is odd.

\section{Proposed BWATEC-Enabled FWBM Architecture}

There is also a need for an FWBM design with an efficient architecture for enabling the proposed BWATEC scheme. Figure 7 describes the hardware architecture of a 16-bit FWBM example enabling the BWATEC functions. As shown in Figure 7, the P.P. values are first produced through the Booth Encoder, and the P.P. Generator operates on two operands of $A$ and $B$, which are already padded with ZP bits according to the prespecified bit width, $L^{\prime}$ of input patterns (Figure 1). Depending on $L^{\prime}$, the BWATEC-associated $\delta_{j}, \gamma$, and $\lambda_{j}$ terms are also set and sent to the P.P. array, along with the P.P. terms. The carry-save adder/carry-propagation adder (CSA/CPA) unit performs the array operations for the $M P, T P_{\text {major }}$ and BWATEC biasing. Right shifting of bits can be optionally executed at the CPA output depending on the practical system design. As detailed in Figure 7, we used four groups (i.e., $\mathrm{M}_{1}, \mathrm{M}_{2}, \mathrm{M}_{3}$, and $\mathrm{M}_{4}$ ) of multiplexers controlled by the setting of $L^{\prime}$ to enable data selection of the carry of $\delta_{j}$ accumulation, $\gamma, \lambda_{j}$, and " 0 " for the BWATEC operations described in Figure 4. A switch is also employed for selecting $\gamma$ or an extra carry contributed by the addition of $p_{0,7}$ and $n_{7}$ for $L^{\prime}=16$. Based on the configuration shown in Figure 7, similar approaches can be used to deduce the FWBM design for other bit widths of the operand. As a result, the BWATEC-enabled FWBM can be realized by using the originally required P.P. elements with additional multiplexers (incl. a switch) and control logics for adjustable TEC operations. 


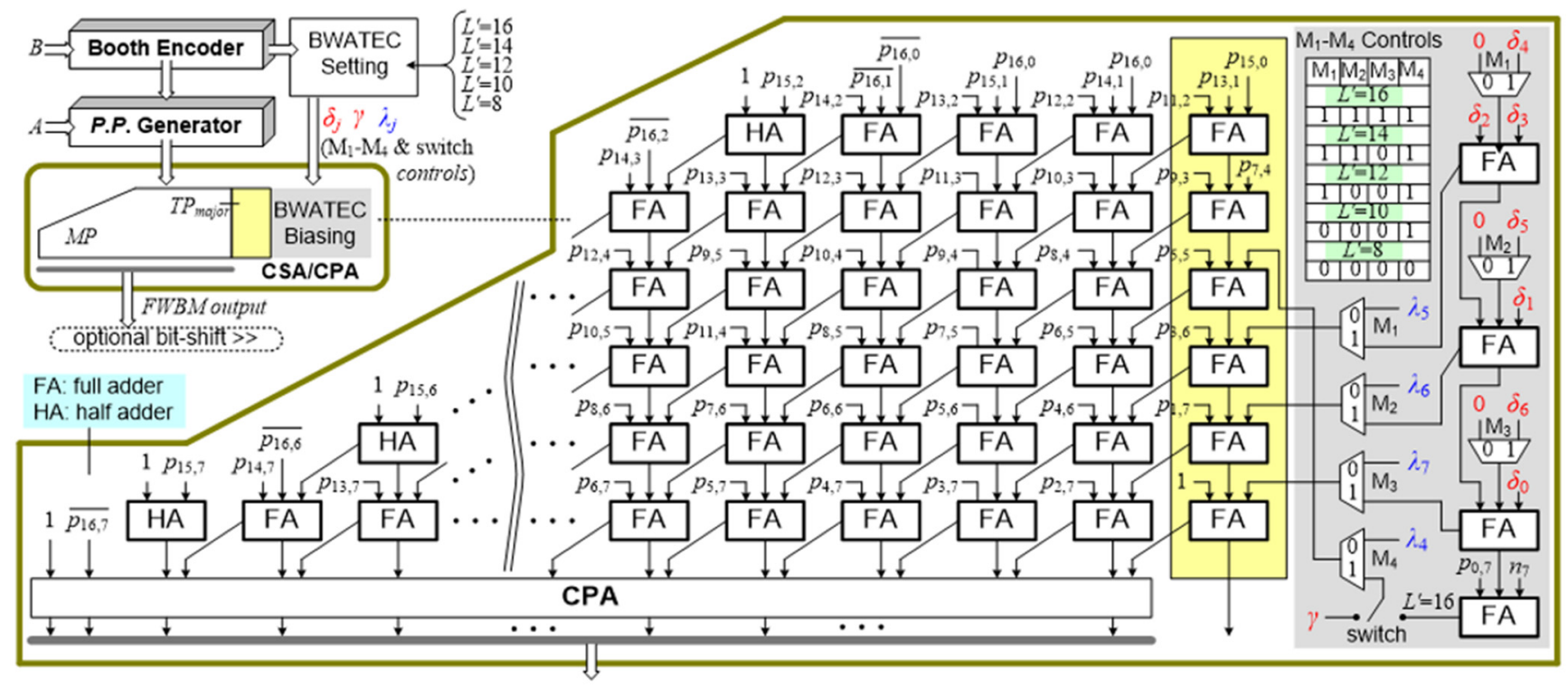

Figure 7. Hardware architecture of 16-bit FWBM that uses the proposed BWATEC scheme.

Considering a general $L$-bit FWBM ( $L$ is scalable) enabling the proposed BWATEC scheme, we see that its hardware configuration for TEC biasing can also be developed based on the BWATEC operation shown in Figure 6, as the approach for the 16-bit FWBM example (refer to Figures 4 and 7). The hardware structure for BWATEC biasing of a general $L$-bit FWBM is described in Figure 8a,b for the two specifications of " $L=2 n$ ( $n$ is even)" and " $L=2 n$ ( $n$ is odd)", respectively. As indicated in Figure 8, the addition of biasing element is performed by using full adders (FAs) or half adders (HAs). The mandatory multiplexers (i.e., MUX1 in Figure 8) are employed to select the carry of $\delta$ accumulation or the $\gamma$ and $\lambda$ terms based on the BWATEC operations in Figure 6, and the optional multiplexers (i.e., MUX2) can allow unadded $\delta$ terms to be " 0 " for energy efficiency. If the devised FWBM is specified to process $L^{\prime}$-bit inputs with small $L^{\prime}$ values, corresponding levels of multiplexers (i.e., MUX3) might be employed to mask the uncalculated $\gamma$ or $\lambda$ terms (refer to Figure 6) as shown in Figure 8. Based on the configuration of a P.P. array and the BWATEC biasing (refer to Figure 8), the hardware (HW) resource usage in the number of FAs, HAs, and multipliers (i.e., MUX1, 2, and 3) of a general $L$-bit FWBM using the proposed BWATEC scheme for various $L^{\prime}$-bit inputs are listed in Table 4.

Table 4. HW resources usage of a general $L$-bit FWBM for various $L^{\prime}$-bit inputs using the BWATEC scheme $(Q=L / 2)$.

\begin{tabular}{cccccc}
\hline MP/TP & major \\
\hline \#W Resources & \multicolumn{4}{c}{ BWATEC Biasing HW Resources } \\
\hline \multirow{2}{*}{$\frac{Q}{2} \times(4+L)+(Q-1)$} & \#FA/HA & \#MUX1 & \#MUX2 & \#MUX3 \\
\hline & $L=2 n($ even $n)$ & $Q / 2$ & $Q / 2$ & $\lceil Q / 2-1\rceil$ & $(1 / 2) \times\left(L / 2-L^{\prime}\right)$ \\
\hline
\end{tabular}




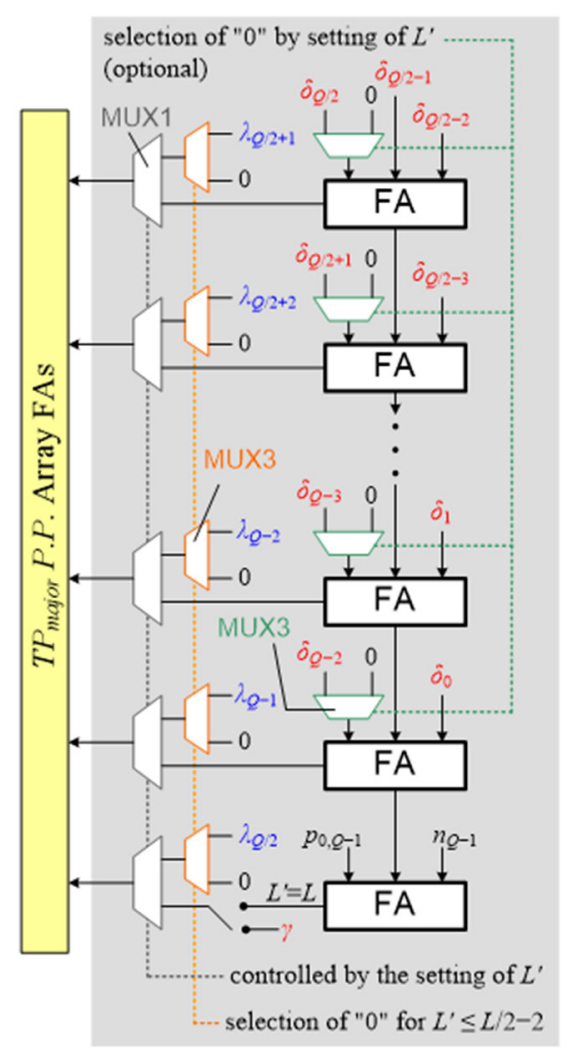

(a)

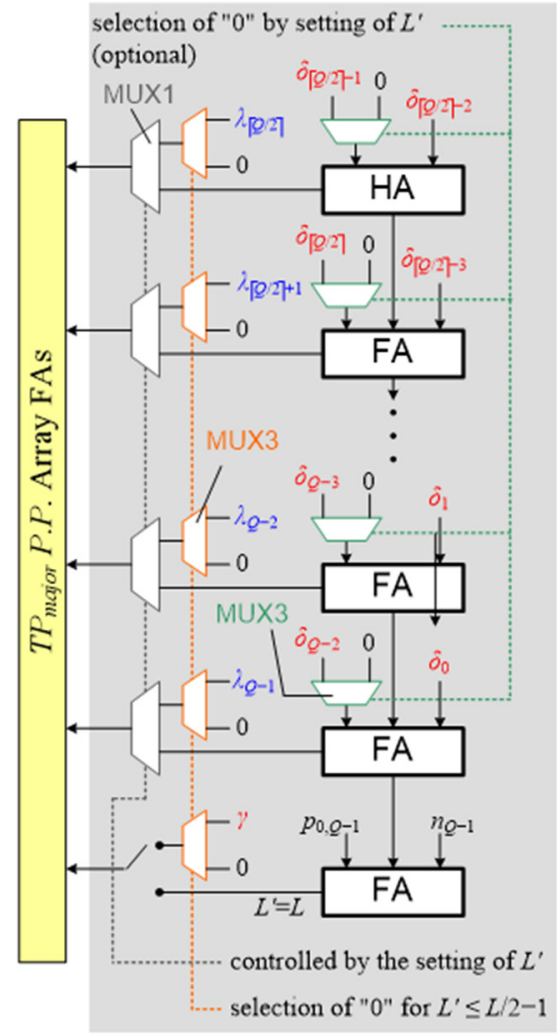

(b)

Figure 8. Hardware configuration for BWATEC biasing of a general $L$-bit FWBM: (a) $L=2 n$, and $n$ is even; (b) $L=2 n$, and $n$ is odd.

\section{Evaluations and Experiments}

Considering 16-bit FWBM designs with TEC based on one-MSC TP $P_{\text {major }}$, this section evaluates the accuracy and hardware performances for the proposed design and several representative works in previous studies. Moreover, the 16-bit FWBM with BWATEC was verified through the SoC-FPGA implementation for CNN inference operations.

\subsection{Evaluations of Accuracy and Hardware Performances}

For the accuracy performance, the signal-to-noise ratio (SNR) is the most important parameter and is defined as in Equation (13), where $F P$ (refer to Equation (3)) is the product of the full-width Booth multiplier, and $F P_{q}$ (refer to Equation (4)) is the product of the FWBM with TEC, DT, or PT. In Equation (12), the mean square error (MSE) is also defined.

$$
S N R(\mathrm{~dB})=10 \times \log _{10}\left(E\left[F P^{2}\right] / E\left[\left(F P-F P_{q}\right)^{2}\right]\right) ; M S E=E\left[\left(F P-F P_{q}\right)^{2}\right] / 2^{2 L}
$$

For comparison, we select state-of-the-art TEC schemes whose functions have a closed form, i.e., the generalized probabilistic estimation bias (GPEB) [16,17], probability estimation and computer simulation (PACS) [20], Booth-encoded sign-digit-based conditional probability (BSCP) [22], and SC-generator-based (SCG) [12] methods, as well as the DT and PT approaches. Table 5 presents the accuracy (i.e., the SNR) and hardware performances (area, critical-path delay, and power consumption) for a 16-bit FWBM using the aforementioned TEC and proposed BWATEC schemes, respectively. 
Table 5. Comparison of accuracy and hardware performances of a 16-bit fixed-width Booth multiplier (FWBM) for PT, DT, and various truncation error compensation (TEC) schemes.

\begin{tabular}{cccccccc}
\hline & PT & BSCP & PACS & GPEB & SCG & Ours & DT \\
\hline \multicolumn{7}{c}{ Accuracy } & Performance $\left(L^{\prime}=16\right)-S N R(\mathrm{~dB})$ \\
\hline SNR & 85.56 & 81.91 & 81.83 & 79.34 & 81.84 & 81.87 & 64.84 \\
\hline Hrea & 2294 & 1330 & 1301 & 1249 & 1325 & 1312 & 1098 \\
\hline Delay & 3.62 & 3.25 & 3.24 & 3.20 & 3.24 & 3.27 & 2.96 \\
\hline Power & 1075 & 615.1 & 585.2 & 562.3 & 612.9 & 591.0 & 486.4 \\
\hline
\end{tabular}

In Table 5, the SNR results were obtained for operations of 16-bit data (i.e., $L^{\prime}=16$ ), based on the calculation of $30 \mathrm{~K}$ sets of 16-bit $A \times B$ Booth multiplication. Both the $A$ and $B$ operands were uncorrelated random 16-bit numbers with uniform distribution in statistics. The hardware parameters were provided by using the Synopsys Design Compiler, through logic synthesis with the TSMC $40 \mathrm{~nm}$ typical standard cell library for FWBM designs with no optional bit-shift processing at the output. According to References [12,22], we used a general sorting circuit based on Reference [12] for the BSCP and SCG designs in order to avoid the addition of negative digit values. In Table 5, the BSCP method achieves a better SNR than all other TEC-enabled designs. However, this result of the BSCP approach was obtained by using a complex TEC formula (i.e., Equation (19) in Reference [22]), and this function is difficult to be directly applied to a practical biasing circuit. The GPEB scheme outperforms other TEC-based works due to its use of a simple 1- bit or 2-bit constant TEC bias; however, the GPEB accuracy result is comparatively more reduced. Referring to the hardware parameters listed in Table 5, the results from the "area" and "power" items basically exhibit the same trend. To benchmark both the area efficiency and the accuracy, a design metric of area-delay-error product $(A D E P)$, defined as "ADEP $=$ Area $\times$ Delay $\times M S E^{\prime \prime}$, can be adopted to evaluate the overall design efficiency. As there is no TEC function involved in either DT or PT and the MSE magnitudes obtained by DT and PT are too extreme for the $A D E P$ evaluation, these two schemes are excluded from the $A D E P$ evaluation [22]. Table 6 lists the $A D E P$ results in percentage values (normalized to that of the GPEB case) for TEC-enabled designs.

Table 6. Comparison of $A D E P$ results of a 16-bit FWBM for various TEC schemes.

\begin{tabular}{cccccc}
\hline & BSCP & PACS & GPEB & SCG & Ours \\
\hline$A D E P(\%)$ & $59.80 \%$ & $59.35 \%$ & $100 \%$ & $60.32 \%$ & $59.71 \%$ \\
\hline
\end{tabular}

As indicated in Table 6, the proposed design outperforms all listed schemes (i.e., a relatively small $A D E P$ value) except the PACS method. This is because additional dataselection multiplexers/switch and control logics are required in our design to enable the adjustment of the TEC function for multiple $L^{\prime}$ levels (refer to Figure 7). Such processing increases hardware costs and especially increases critical-path delay in our FWBM relative to other TEC designs, as shown in Table 5; however, the accuracy for $L^{\prime}=16$ in our case is comparatively improved by using deterministic $p_{0,7}$ and $n_{7}$ (refer to Section 3.2, Figure 4).

Nevertheless, the actual accuracy performance and hardware efficiency of the proposed design is manifested in the accuracy improvement for operations on $L^{\prime}$-bit input patterns, giving $L^{\prime}<16$. Table 7 reports the SNR results for the TEC-enabled 16-bit FWBMs (i.e., works in Table 6) for operations of $L^{\prime}=14,12,10$, and 8. In Table 7, the SNR values were obtained based on the 16-bit product of FWBMs relative to the PT outcomes. As indicated in Table 7, our design achieves the highest SNR performances compared to other TEC-based designs for all listed $L^{\prime}$ cases because the proposed BWATEC scheme provides 
more precise TEC biasing for various $L^{\prime}$-bit inputs. In addition, higher $S N R$ results can be achieved with smaller $L^{\prime}$ values by using the proposed design, due to more counts of deterministic $R_{Z} / R_{D}$ elements. In practical designs, a slight improvement in the SNR results possibly results in an efficient enhancement in the system operation accuracy [21].

Table 7. SNR results (dB) of a 16-bit FWBM for various TEC schemes and $L^{\prime}$-bit inputs.

\begin{tabular}{cccccc}
\hline & BSCP & PACS & GPEB & SCG & Ours \\
\hline$L^{\prime}=14$ & 80.62 & 81.06 & 78.10 & 81.04 & 81.69 \\
\hline$L^{\prime}=12$ & 80.77 & 81.25 & 76.67 & 80.96 & 82.12 \\
\hline$L^{\prime}=10$ & 81.01 & 81.57 & 75.40 & 81.28 & 83.18 \\
\hline$L^{\prime}=8$ & 80.14 & 81.36 & 74.65 & 80.38 & Inf. \\
\hline
\end{tabular}

Considering the overall design efficiency, Figure 9 illustrates the $A D E P$ results from the TEC-based 16-bit FWBMs based on the MSE value relative to the PT products for operations of $L^{\prime}=14,12,10$, and 8, with ZP bits added to the input operand. In Figure 9, the $A D E P$ values are normalized to the GPEB results, and the annotated percentage values represent the reduction of the $A D E P$ achieved by the proposed design relative to all other listed methods. Figure 9 demonstrates that our scheme outperforms its contenders in terms of the $A D E P$ values, achieving reductions of $7.9-50.9 \%, 17.1-69.5 \%, 29.9-82.2 \%$, and $100 \%$ for the operations of input patterns with 14-bit, 12-bit, 10-bit, and 8-bit widths, respectively. Figure 9 shows that our design can achieve a more significant TEC effect with smaller specified $L^{\prime}$ values, as more ratios of deterministic values are used and associated with the $R_{H}$ and $R_{D}$ when using the proposed BWATEC scheme. For the case of $L^{\prime}=8$, our approach equivalently counts all P.P. terms to obtain a full-width result that is the same as a PT outcome, and thus a $100 \% A D E P$ reduction can be achieved.

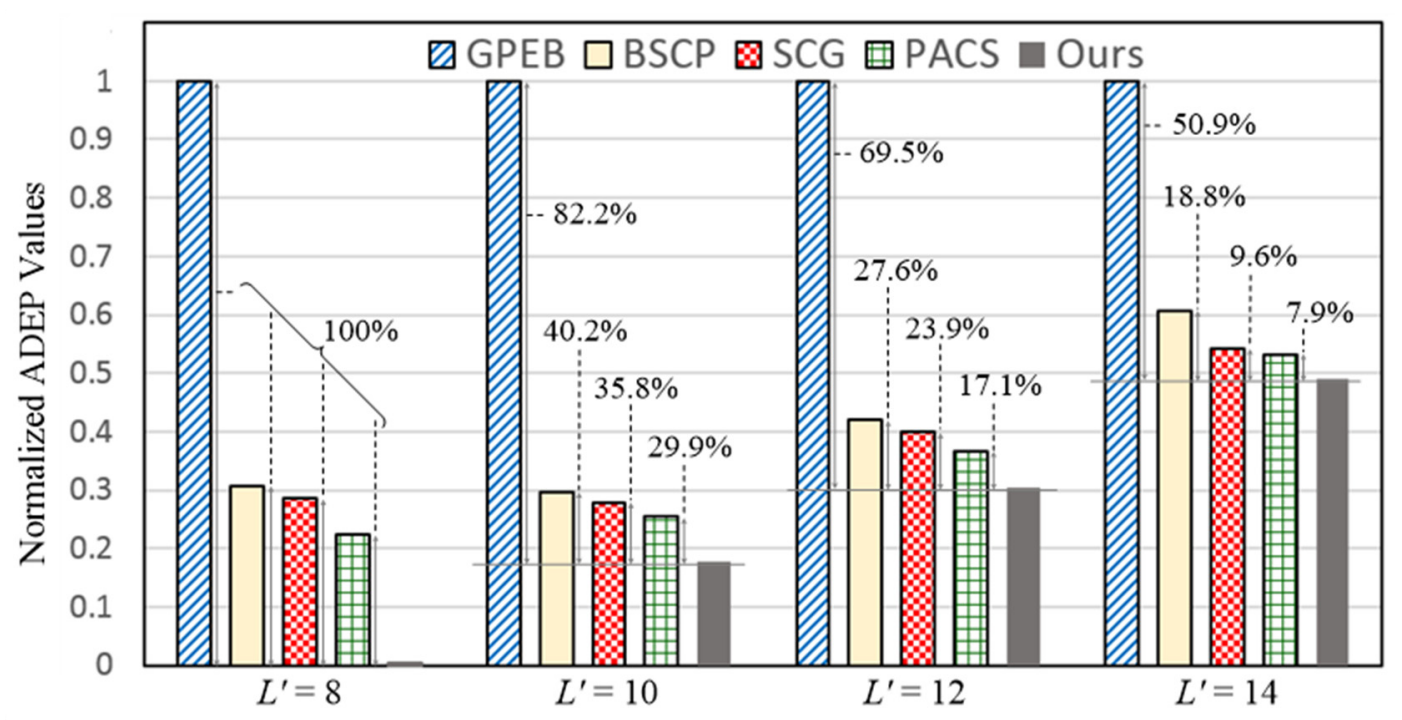

Figure 9. Normalized $A D E P$ values of a 16-bit FWBM for various TEC schemes and $L^{\prime}$.

As discussed in Sections 3.3 and 4, two specifications of " $L=2 n$ ( $n$ is even)" and " $L=2 n$ ( $n$ is odd)" are considered for the design scalability of an L-bit FWBM, using the proposed BWATEC scheme. Therefore, in addition to the case of $L=16$ (for even $n$ ), another case of $L=14$ (for odd $n$ ) was also evaluated for the $A D E P$ performances in this section. Figure 10 illustrates the $A D E P$ results from the TEC-enabled 14-bit FWBMs for operations of $L^{\prime}=12$, 10,8 , and 6, based on the same processing with that for the 16-bit FWBM evaluation. Figure 10 indicates that our design outperforms all other listed methods, achieving the significant $A D E P$ reductions for the operations of inputs with 12-bit, 10-bit, 8-bit, and 6-bit 
widths, respectively. Compared to the $A D E P$ results for 16-bit FWBMs (Figure 9), the ADEP drops for all GPEB-excluded designs in relation to the GPEB base is reduced in Figure 10; however, the same trend of the $A D E P$ reductions based on the relative value of $L$ and $L^{\prime}$ is exhibited for our design associated with other TEC-based works.

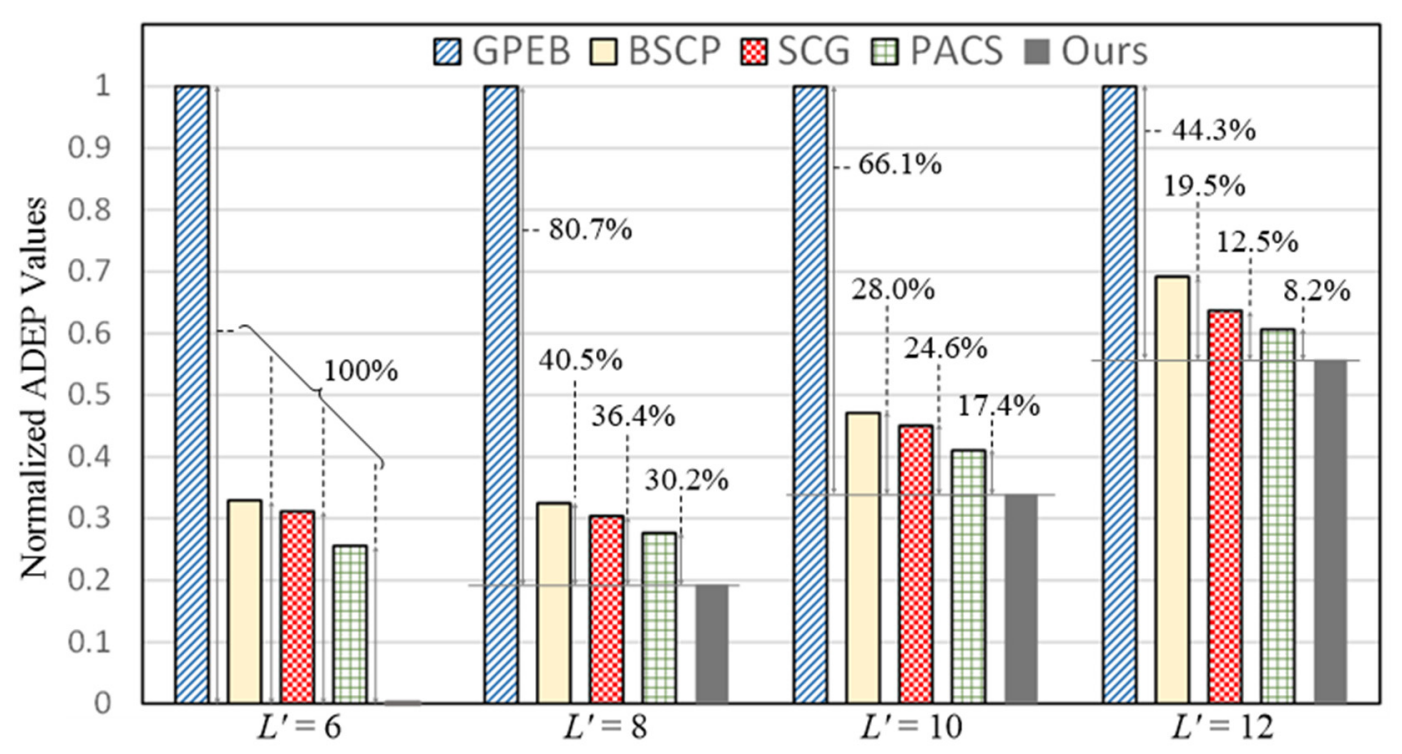

Figure 10. Normalized $A D E P$ values of a 14-bit FWBM for various TEC schemes and $L^{\prime}$.

\subsection{Design Verification and Experiments}

\subsubsection{CNN Acceleration Application}

To verify an FWBM enabling the proposed BWATEC scheme, we implemented our design by using a SoC-FPGA platform and demonstrated the hardware acceleration for CNN inference operations. In a typical CNN accelerator, fixed-point operations are usually considered and a suitable bit width can be determined based on the CNN inference accuracy requirement $[25,26]$. Several studies have shown that the small bit width (e.g., 8-bit width or fewer) is sufficient for the model coefficients and operation precision, while preserve the inference accuracy [27-29]. However, a sufficiently high bit width (e.g., common 16 bits) is considered in several $\mathrm{CNN}$ accelerator approaches to ensure the precision required by various applications [30-32]. Moreover, different bit-widths cab be specified for different CNN layers (e.g., the intermediate layers) to adjust the CNN performance [33,34]. Accordingly, several works have proposed $\mathrm{CNN}$ processing units that support operations with variable bit widths (e.g., 4/8/16-bit or 1-bit to 16-bit) [28,35-37]. In this study, an L-bit FWBM (e.g., our design example of a 16-bit FWBM) capable of processing input patterns with multiple $L^{\prime}$-bit $\left(L^{\prime} \leq L\right)$ widths lends support to the aforementioned practical approaches.

\subsubsection{SoC-FPGA Implementation}

The employed SoC-FPGA-based platform uses a Xilinx Zynq-7000 SoC-FPGA device which integrates an ARM central processing unit (CPU) with the user-developed hardware side. Such a SoC-FPGA approach lends support to the CNN inference operations by using a software (SW)-hardware (HW) co-design scheme [38-40] by appropriately evaluating the SW-HW work division. For example, computation-expensive two-dimensional (2D) convolution is often accelerated at the HW side, while other low-effort CNN operations, such as maximum pooling, fully-connected (FC) layer execution and system controls are processed at the SW end [39,40]. Figure 11 shows the setup of our implementation that uses a SoC-FPGA approach based on the SW-HW co-design for CNN acceleration. 


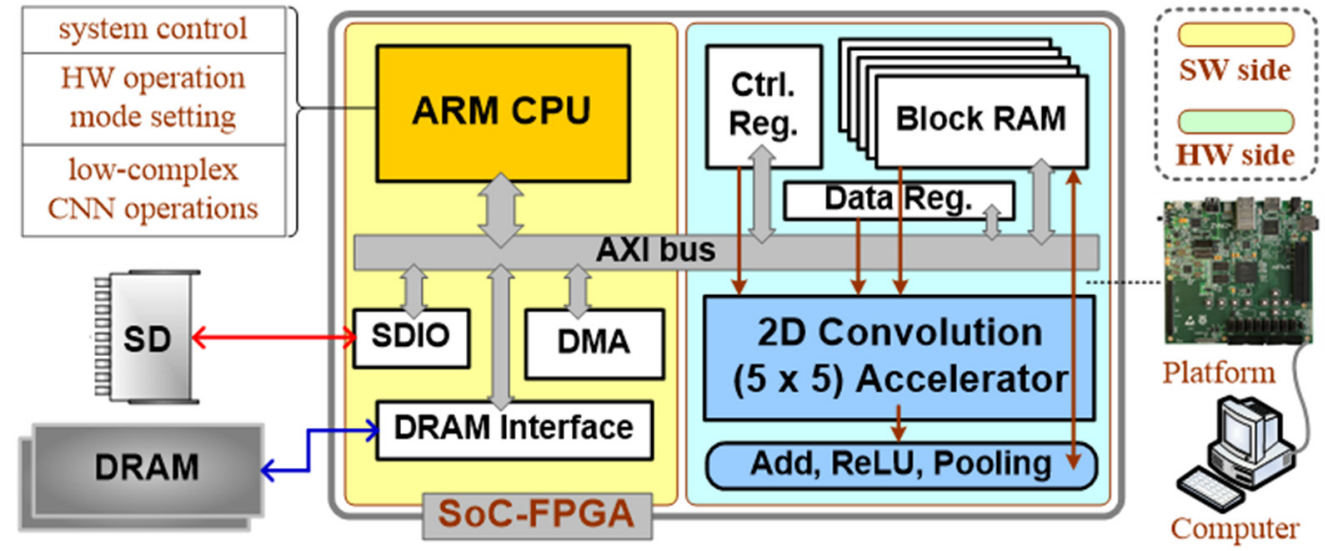

Figure 11. Schematic of the setup of our design implementation that uses a SoC-FPGA approach.

Referring to Figure 11, the division of HW and SW responsibilities in our CNN setup was as follows. The HW side was responsible for $2 \mathrm{D}(5 \times 5)$ convolution, addition of an offset, activation function (i.e., rectified linear unit; ReLU), and maximum pooling, while the SW side performed residual low-complex operations (e.g., FC execution), HW operation mode setting, and system control. As depicted in Figure 11, the ARM CPU executes SW commands and communicates with the HW side through the AXI bus, and the data transferring between the external memory and the FPGA HW-side memory is executed through direct memory access (DMA). When the HW acceleration of each CNN layer was actuated, the feature map data, kernel weights, and control parameters were fetched from the external memory (e.g., DRAM) to the HW side via DMA transmission and stored in the block RAMs, data registers, and control registers, respectively. The $2 \mathrm{D}$ convolution accelerator then accessed those stored values for convolution operations and then sent the calculated result to the next module for the offset-addition, ReLU, and pooling operations. The final produced data of $\mathrm{HW}$ acceleration for each $\mathrm{CNN}$ layer were stored in the block RAMs and sent to the external memory through DMA for the follow-up SW processing.

In our design, the $2 \mathrm{D}(5 \times 5)$ convolution accelerator employs 25 16-bit FWBMs with BWATEC, which can operate with multiple 16-bit, 14-bit, 12-bit, 10-bit, or 8-bit numerical input data. Depending on the tilling for each CNN layer, the block RAMs can be configured to store the data of input and output feature maps (images) with sizes from $32 \times 32$ to $128 \times 128$. Table 8 lists the main HW resource usage on a Xilinx/Zynq-7000 SoC-FPGA device for our FPGA design, and the items include the lookup-table (LUT), flip-flop (FF), LUTRAM, and block RAM (BRAM)utilization. Table 8 also lists the HW performance of giga operations per second (GOPs), which is obtained by using a $50 \mathrm{MHz}$ clock rate with values converted from the giga multiplication and addition operations [40].

Table 8. HW resources and performances for our FPGA implementation.

\begin{tabular}{ccccc}
\hline LUT Util. & LUTRAM Util. & FF Util. & BRAM Util. & GOPs \\
\hline 6786 & 62 & 2572 & 12.5 & 2.55 \\
\hline
\end{tabular}

\subsubsection{Electrocardiogram Classification Experiment}

Based on our SoC-FPGA implementation and SW-HW co-design setup, an experiment was performed to demonstrate the electrocardiogram (ECG) classification. In this work, we used the standard MIT-BIH arrhythmia dataset [41] for the CNN model training and inference. To operate with ECG data by using a 2D CNN model [42,43], we transformed the one-dimensional MIT-BIH ECG signals into the $(128 \times 128) 2$ D ECG image by using the signal preprocessing technique presented in Reference [42]. Rather than the clinical ECG classification for seven or five arrhythmia classes [42,44], our experiment 
merely classified ECG images into "normal" and "abnormal" heartbeats for wearable ECG monitor applications.

The experimental network for the aimed ECG classification was built up by using a simplified LeNet-5 CNN model [45]. As the contents summarized in Table 9, the built-up CNN model includes two convolution and maximum pooling layers, followed by the FC layers. Our CNN model was first determined by training process performed on a high-end computer in floating-point operations. For the CNN inference using an SW-HW co-design approach, the executions accelerated at the HW side were performed in fixedpoint operations to achieve available overall accuracy (Table 9). To verify the proposed design, we implemented a 2D convolution unit consisting of 25 pcs 16-bit FWBMs with the proposed BWATEC function on the SoC-FPGA device. In our experiment, the same 2D convolution unit with one set of 16-bit FWBMs ( 25 pcs) was operated to perform the computation of two CNN layers. To demonstrate the BWATEC operations of our design, two phases of $L^{\prime}$ setting for the same set of 25 pcs 16-bit FWBMs (i.e., $L=16$; $L^{\prime}$-bit inputs) were adopted to execute two layers of $\mathrm{CNN}$ convolution execution. In phase 1, all 16-bit FWBMs in the 2D convolution unit were set to operate with 12-bit input data (i.e., $L^{\prime}=12$ ) for $\mathrm{CNN}$ layer-1 operations to consist with the numerical level of inputs. In phase 2, the same 16-bit FWBMs were set to process 16-bit input data (i.e., $L^{\prime}=16$ ) for CNN layer-2 operations to preserve the computation precision.

Table 9. Experimental CNN model architecture and SNR performances among layers.

\begin{tabular}{ccccc}
\hline Layers & $\begin{array}{c}\text { Input Feature } \\
\text { Map Size }\end{array}$ & $\begin{array}{c}\text { Input } \\
\text { Channel No. }\end{array}$ & Kernel Size & $\begin{array}{c}\text { Output } \\
\text { SNR (dB) }\end{array}$ \\
\hline Input & $128 \times 128$ & 1 & - & - \\
\hline 1st Convolution & $128 \times 128$ & 1 & $5 \times 5$ & 34.37 \\
\hline 1st Max. Pooling & $128 \times 128$ & 4 & $2 \times 2$ & 35.14 \\
\hline 2nd Convolution & $64 \times 64$ & 4 & $5 \times 5$ & 29.48 \\
\hline 2nd Max. Pooling & $64 \times 64$ & 8 & $2 \times 2$ & 29.86 \\
\hline FC & $32 \times 32$ & 8 & - & - \\
\hline
\end{tabular}

For evaluation, we also implemented contrast 2D convolution units composed of 16bit FWBMs, using the BSCP, PACS, GPEB, and SCG TEC schemes on the same SoC-FPGA device. Table 10 lists the FPGA LUT resource utilization for a 2D convolution unit with various TEC-based FWBM designs, using the aforementioned methods and our scheme. As shown in Table 10, our design achieves the medium level of area efficiency on FPGA, which is basically consist with the trend of area parameters listed in Table 7. However, the feature of our design for multiple setting of $L^{\prime}$-bit operations lends support to the system development requiring flexible word lengths or improved accuracy. For a case study in addition to CNN acceleration, the devised 2D convolution unit can be restructured to realize a 25-tap finite impulse response (FIR) filter by inserting several multiplexers in the data paths of 25 pcs FWBMs. We also developed such an FIR with a slight HW overhead via FPGA implementation to use our design for digital signal processing applications.

Table 10. Comparison of lookup-table (LUT) resource usage for FPGA implementation of a 2D convolution unit for various TEC schemes.

\begin{tabular}{cccccc}
\hline & BSCP & PACS & GPEB & SCG & Ours \\
\hline LUT Util. & 5225 & 4907 & 4459 & 5206 & 4957 \\
\hline
\end{tabular}

The ECG classification was checked by using a modified CNN inference model with the $2 \mathrm{D}$ convolution performed in our two-phase fixed-point operations. Moreover, the 
experimental CNN operations (Table 9) were performed by using the SoC-FPGA based on our SW-HW co-design approach to obtain the inference results. For the bit-width setting (i.e., $L^{\prime}$ ) of two phases, the SW side would prepare the FWBM operands appropriately padded with ZP bits and set the BWATEC control for each round (i.e., layer 1 or 2) of CNN HW acceleration. The inference outcomes generated via the SoC-FPGA were further compared with the results generated by using the aforementioned fixed-point-operated $\mathrm{CNN}$ inference model for verification. After inference checking, the confusion matrix and performances of our ECG classification experiment are listed in Table 11. The performance results reported in Table 11 were obtained based on the accuracy $(A c c$.$) , sensitivity (\operatorname{Sen}$.$) ,$ and specificity $(S p c$.) statistical metrics extracted from the confusion matrix [42,44]. The terms of TP, TN, FP, and FN denote true positive as "abnormal" (arrhythmia), true negative as "normal", false positive as "abnormal", and false negative as "normal" in the binary classification, respectively. The associated formulas are defined as follows:

$$
\text { Acc. }=\frac{\mathrm{TP}+\mathrm{TN}}{\mathrm{TP}+\mathrm{TN}+\mathrm{FP}+\mathrm{FN}} \text { Sen. }=\frac{\mathrm{TP}}{\mathrm{TP}+\mathrm{FN}} S p c .=\frac{\mathrm{TN}}{\mathrm{TN}+\mathrm{FP}}
$$

Table 11. Confusion matrix and performances of the experimental CNN for ECG classification.

\begin{tabular}{|c|c|c|c|}
\hline${ }_{\text {Label } \downarrow}$ Prediction $\rightarrow$ & Abnormal & Normal & Metrics \\
\hline Abnormal & 27,577 (TP) & $705(\mathrm{FN})$ & 97.5\% (Sen.) \\
\hline Normal & $2266(\mathrm{FP})$ & $35,316(\mathrm{TN})$ & $94.0 \%(S p c)$. \\
\hline Metrics & \multicolumn{2}{|c|}{$95.5 \%(A c c)}$. & \\
\hline
\end{tabular}

\section{Conclusions}

In this paper, we presented a BWATEC scheme capable of providing an adjusted TEC function adaptive to various $L^{\prime}$-bit input patterns of an $L$-bit FWBM, in which $L^{\prime} \leq L$. Using different combinations of hybrid deterministic/probabilistic values associated with the $\mathrm{R}_{\mathrm{H}}$ and $\mathrm{R}_{\mathrm{D}}$ regions, the proposed BWATEC scheme can generate a tailored high-accuracy TEC bias for an $L$-bit FWBM, depending on the setting of $L^{\prime}$ ( $L$ and $L^{\prime}$ are scalable). An FWBM enabling the proposed BWATEC scheme can be realized by using a reconfigurable bias circuit in a P.P. array with design scalability.

Taking a 16-bit FWBM as an example, we found that the approach using our BWATEC scheme exhibited design efficiency and different degrees of $A D E P$ reduction for operations with 14-bit to 8-bit inputs, as compared to FWBM designs that used state-of-the-art TEC methods.

Moreover, the resultant 16-bit FWBM with BWATEC were verified by using the Xilinx Zynq-7000 SoC-FPGA based on the SW-HW co-design approach. The SoC-FPGA-based verification demonstrated the experimental CNN model for ECG classification.

Author Contributions: Conceptualization and methodology, S.-N.T.; review and editing, S.-N.T.; project administration, S.-N.T.; software and validation (experiment), J.-C.L., C.-K.C., P.-T.K. and Y.-S.C. All authors have read and agreed to the published version of the manuscript.

Funding: This work was supported in part by the Ministry of Science and Technology of Taiwan, under project MOST 110-2221-E-033-039.

Informed Consent Statement: C-language simulation and Verilog modeling supports. We also thank Hong-Yu Ke and Jia-Nan Zhong for their works in the CNN model development for ECG classification.

Acknowledgments: The authors thank Yu-Shin Han and Jih-Hsiang Yeh for their works.

Conflicts of Interest: The authors declare no conflict of interest. 


\section{References}

1. Parhi, K.K. VLSI Digital Signal Processing Systems: Design and Implementation, 1st ed.; Wiley: New York, NY, USA, 1999.

2. Lee, H.Y.; Park, I.C. Balanced Binary-Tree Decomposition for Area-Efficient Pipelined FFT Processing. IEEE Trans. Circuits Syst. I Reg. Pap. 2007, 54, 889-900. [CrossRef]

3. Chen, H.Y.; Lin, J.N.; Hu, H.S.; Jou, S.J. STBC-OFDM Downlink Baseband Receiver for Mobile WMAN. IEEE Trans. Very Large Scale Integr. (VLSI) Syst. 2013, 21, 43-54. [CrossRef]

4. Tang, S.-N.; Han, Y.-S. A High-Accuracy Hardware-Efficient Multiply-Accumulate (MAC) Unit Based on Dual-Mode Truncation Error Compensation for CNNs. IEEE Access 2020, 8, 214716-214731. [CrossRef]

5. Van, L.-D.; Yang, C.-C. Generalized Low-Error Area-Efficient Fixed-Width Multipliers. IEEE Trans. Circuits Syst. I Reg. Pap. 2005, 52, 1608-1619. [CrossRef]

6. Tu, J.-H.; Van, L.-D. Power-efficient pipelined reconfigurable fixed-width Baugh-Wooley multipliers. IEEE Trans. Comput. 2009, 58, 1346-1355. [CrossRef]

7. Chang, C.-H.; Satzoda, R.K. A Low Error and High Performance Multiplexer-Based Truncated Multiplier. IEEE Trans. Very Large Scale Integr. (VLSI) Syst. 2010, 18, 1767-1771. [CrossRef]

8. Petra, N.; Caro, D.D.; Garofalo, V.; Napoli, E.; Strollo, A.G.M. Design of Fixed-Width Multipliers with Linear Compensation Function. IEEE Trans. Circuits Syst. I Reg. Pap. 2011, 58, 947-960. [CrossRef]

9. Jou, S.-J.; Tsai, M.-H.; Tsao, Y.-L. Low-error reduced-width Booth multipliers for DSP applications. IEEE Trans. Circuits Syst. I Fundam. Theory Appl. 2003, 50, 1470-1474. [CrossRef]

10. Chen, Y.-H.; Chang, T.-Y.; Jou, R.-Y. A statistical error-compensated Booth multipliers and its DCT applications. In Proceedings of the TENCON IEEE Region 10 Conference, Fukuoka, Japan, 21-24 November 2010; pp. 1146-1149. [CrossRef]

11. Song, M.A.; Van, L.D.; Kuo, S.Y. Adaptive low-error fixed-width Booth multipliers. IEICE Trans. Fundam. Electron. Commun. Comput. Sci. 2007, 90, 1180-1187. [CrossRef]

12. Wang, J.-P.; Kuang, S.-R.; Liang, S.-C. High-Accuracy Fixed-Width Modified Booth Multipliers for Lossy Applications. IEEE Trans. Very Large Scale Integr. (VLSI) Syst. 2011, 19, 52-60. [CrossRef]

13. Kuang, S.-R.; Wang, J.-P.; Guo, C.-Y. Modified Booth Multipliers with a Regular Partial Product Array. IEEE Trans. Circuits Syst. II Exp. Briefs 2009, 56, 404-408. [CrossRef]

14. Cho, K.-J.; Lee, K.-C.; Chung, J.-G.; Parhi, K.K. Design of low-error fixed-width modified Booth multiplier. IEEE Trans. Very Large Scale Integr. (VLSI) Syst. 2004, 12, 522-531. [CrossRef]

15. Juang, T.-B.; Hsiao, S.-F. Low-error carry-free fixed-width multi- pliers with low-cost compensation circuits. IEEE Trans. Circuits Syst. II Exp. Briefs 2005, 52, 299-303. [CrossRef]

16. Li, C.-Y.; Chen, Y.-H.; Chang, T.-Y.; Chen, J.-N. A Probabilistic Estimation Bias Circuit for Fixed-Width Booth Multiplier and Its DCT Applications. IEEE Trans. Circuits Syst. II Exp. Briefs 2011, 58, 215-219. [CrossRef]

17. Chen, Y.-H.; Li, C.-Y.; Chang, T.-Y. Area-Effective and Power-Efficient Fixed-Width Booth Multipliers Using Generalized Probabilistic Estimation Bias. IEEE J. Emerg. Sel. Top. Circuits Syst. 2011, 1, 277-288. [CrossRef]

18. Chen, Y.-H.; Chang, T.-Y. A High-Accuracy Adaptive Conditional Probability Estimator for Fixed-Width Booth Multipliers. IEEE Trans. Circuits Syst. I Reg. Pap. 2012, 59, 594-603. [CrossRef]

19. Chen, Y.-H. An Accuracy-Adjustment Fixed-Width Booth Multiplier Based on Multilevel Conditional Probability. IEEE Trans. Very Large Scale Integr. (VLSI) Syst. 2015, 23, 203-207. [CrossRef]

20. He, W.-Q.; Chen, Y.-H.; Jou, S.-J. High-Accuracy Fixed-Width Booth Multipliers Based on Probability and Simulation. IEEE Trans. Circuits Syst. I Reg. Pap. 2015, 62, 2052-2061. [CrossRef]

21. Chen, Y.-H. Improvement of Accuracy of Fixed-Width Booth Multipliers Using Data Scaling Technology. IEEE Trans. Circuits Syst. II Exp. Briefs 2021, 68, 1018-1022. [CrossRef]

22. Zhang, Z.; He, Y. A Low-Error Energy-Efficient Fixed-Width Booth Multiplier with Sign-Digit-Based Conditional Probability Estimation. IEEE Trans. Circuits Syst. II Express Briefs 2018, 65, 236-240. [CrossRef]

23. Oklobdzija, V.G.; Villeger, D.; Liu, S.S. A method for speed optimized partial product reduction and generation of fast parallel multipliers using an algorithmic approach. IEEE Trans. Comput. 1996, 45, 294-306. [CrossRef]

24. He, Y.; Chang, C.-H. A New Redundant Binary Booth Encoding for Fast $2^{\mathrm{n}}$-Bit Multiplier Design. IEEE Trans. Circuits Syst. I Reg. Pap. 2009, 56, 1192-1201. [CrossRef]

25. Gong, L.; Wang, C.; Li, X.; Chen, H.; Zhou, X. MALOC: A Fully Pipelined FPGA Accelerator for Convolutional Neural Networks with All Layers Mapped on Chip. IEEE Trans. Comput. Aided Des. Integr. Circuits Syst. 2018, 37, 2601-2612. [CrossRef]

26. Zhang, L.; Li, B.; Liu, Y.; Zhao, X.; Wang, Y.; Wu, J. FPGA Acceleration of CNNs-Based Malware Traffic Classification. Electronics 2020, 9, 1631. [CrossRef]

27. Moons, B.; Verhelst, M. An Energy-Efficient Precision-Scalable ConvNet Processor in 40-nm CMOS. IEEE J. Solid-State Circuits 2017, 52, 903-914. [CrossRef]

28. Camus, V.; Mei, L.; Enz, C.; Verhelst, M. Review and Benchmarking of Precision-Scalable Multiply-Accumulate Unit Architectures for Embedded Neural-Network Processing. IEEE J. Emerg. Sel. Top. Circuits Syst. 2019, 9, 697-711. [CrossRef]

29. Chen, Q.; Fu, Y.; Song, W.; Cheng, K.; Lu, Z.; Zhang, C.; Li, L. An Efficient Streaming Accelerator for Low Bit-Width Convolutional Neural Networks. Electronics 2019, 8, 371. [CrossRef] 
30. Chen, Y.; Krishna, T.; Emer, J.S.; Sze, V. Eyeriss: An Energy-Efficient Reconfigurable Accelerator for Deep Convolutional Neural Networks. IEEE J. Solid-State Circuits 2017, 52, 127-138. [CrossRef]

31. Du, L.; Du, Y.; Li, Y.; Su, J.; Kuan, Y.-C.; Liu, C.-C.; Chang, M.-C.F. A Reconfigurable Streaming Deep Convolutional Neural Network Accelerator for Internet of Things. IEEE Trans. Circuits Syst. I Regul. Pap. 2018, 65, 198-208. [CrossRef]

32. Jo, J.; Cha, S.; Rho, D.; Park, I. DSIP: A Scalable Inference Accelerator for Convolutional Neural Networks. IEEE J. Solid-State Circuits 2018, 53, 605-618. [CrossRef]

33. Zhou, S.; Wu, Y.; Ni, Z.; Zhou, X.; Wen, H.; Zou, Y. DoReFa-Net: Training Low Bitwidth Convolutional Neural Networks with Low Bitwidth Gradients. arXiv 2016, arXiv:1606.06160. Available online: https:/ / arxiv.org/abs/1606.06160v2 (accessed on 11 October 2021).

34. Park, E.; Ahn, J.; Yoo, S. Weighted-Entropy-Based Quantization for Deep Neural Networks. In Proceedings of the IEEE Conference on Computer Vision and Pattern Recognition (CVPR), Honolulu, HI, USA, 21-26 July 2017. [CrossRef]

35. Shin, D.; Lee, J.; Lee, J.; Yoo, H.J. DNPU: An 8.1 TOPS/W reconfigurable CNN-RNN processor for general-purpose deep neural networks. In Proceedings of the IEEE Int. Solid-State Circuits Conference (ISSCC), San Francisco, CA, USA, 5-9 February 2017; pp. 240-241. [CrossRef]

36. Garofalo, A.; Tagliavini, G.; Conti, F.; Rossi, D.; Benini, L. XpulpNN: Accelerating Quantized Neural Networks on RISC-V Processors Through ISA Extensions. In Proceedings of the Design, Automation \& Test in Europe Conference \& Exhibition (DATE), Grenoble, France, 9-13 March 2020; pp. 186-191. [CrossRef]

37. Lee, J.; Kim, C.; Kang, S.; Shin, D.; Kim, S.; Yoo, H. UNPU: An Energy-Efficient Deep Neural Network Accelerator with Fully Variable Weight Bit Precision. IEEE J. Solid-State Circuits 2019, 54, 173-185. [CrossRef]

38. Han, Y.; Virupakshappa, K.; Vitor Silva Pinto, E.; Oruklu, E. Hardware/Software Co-Design of a Traffic Sign Recognition System Using Zynq FPGAs. Electronics 2015, 4, 1062-1089. [CrossRef]

39. Guo, K.; Han, S.; Yao, S.; Wang, Y.; Xie, Y.; Yang, H. Software-Hardware Codesign for Efficient Neural Network Acceleration. IEEE Micro 2017, 37, 18-25. [CrossRef]

40. Moini, S.; Alizadeh, B.; Emad, M.; Ebrahimpour, R. A Resource-Limited Hardware Accelerator for Convolutional Neural Networks in Embedded Vision Applications. IEEE Trans. Circuits Syst. II Express Briefs 2017, 64, 1217-1221. [CrossRef]

41. Moody, G.B.; Mark, R.G. The impact of the MIT-BIH arrhythmia database. IEEE Eng. Med. Biol. Mag. 2001, 20, 45-50. [CrossRef]

42. Ju, T.J.; Nguyen, H.M.; Kang, D.; Kim, D.; Kim, D.; Kim, Y.-H. ECG arrhythmia classification using a 2-D convolutional neural network. arXiv 2018, arXiv:1804.06812. Available online: https:/ /arxiv.org/abs/1804.06812 (accessed on 11 October 2021).

43. Wu, Y.; Yang, F.; Liu, Y.; Zha, X.; Yuan, S. A Comparison of 1-D and 2-D Deep Convolutional Neural Networks in ECG Classification. arXiv 2018, arXiv:1810.07088. Available online: https://arxiv.org/abs/1810.07088 (accessed on 11 October 2021).

44. Saadatnejad, S.; Oveisi, M.; Hashemi, M. LSTM-Based ECG Classification for Continuous Monitoring on Personal Wearable Devices. IEEE J. Biomed. Health Inform. 2020, 24, 515-523. [CrossRef]

45. Lecun, Y.; Bottou, L.; Bengio, Y.; Haffner, P. Gradient-based learning applied to document recognition. Proc. IEEE 1998, 86, 2278-2324. [CrossRef] 